\title{
Akute und chronische Durchfallerkrankungen: Differenzialdiagnose und Therapie
}

Carmen Monasterio, Christina Hartl, Peter Hasselblatt

\begin{abstract}
Durchfälle gehören zu den häufigsten in der Hausarztpraxis berichteten Symptomen. Während akute Durchfallerkrankungen in der Regel eine infektiöse Genese haben und spontan ausheilen, kommt es bei der Abklärung chronischer Diarrhöen oft zu Unsicherheiten. Dieser Beitrag soll die Bandbreite möglicher Differenzialdiagnosen sowie die hiermit verbundenen diagnostischen und therapeutischen Herausforderungen akuter und chronischer Durchfallerkrankungen bei Erwachsenen beleuchten.
\end{abstract}

\begin{tabular}{ll}
\hline \multicolumn{2}{l}{ AB KÜRZUNGEN } \\
ATI & Amylase-Trypsin-Inhibitor \\
CAMP & zyklisches Adenosinmonophosphat \\
CED & chronisch entzündliche Darmerkran- \\
& kung \\
CMV & Zytomegalievirus \\
FMT & fäkaler Mikrobiota-Transfer \\
HIV & humanes Immundefizienz-Virus \\
HLA & humanes Leukozyten-Antigen \\
Ig & Immunglobulin \\
IL & Interleukin \\
NSAR & Nonsteroidale Antirheumatika \\
PCR & Polymerase-Kettenreaktion \\
PPI & Protonenpumpeninhibitor \\
RDS & Reizdarmsyndrom \\
Rho-GTPase & Rho homologue GTPase \\
RT-PCR & Reverse-Transkriptase-PCR \\
SIBO & small intestinal bacterial Overgrowth \\
TCdA/TcdB & C. fidd. Toxin A/B \\
TNF & Tumornekrosefaktor \\
VIPom & vasointestinales Peptid \\
ZNS & zentrales Nervensystem \\
& \\
\hline
\end{tabular}

\section{DEFINITION}

Diarrhö wird über eine vermehrte Stuhlfrequenz >3$\mathrm{mal} /$ Tag und verminderte Stuhlkonsistenz (breiig bis flüssig) definiert. Hiervon abzugrenzen sind vermeintliche Durchfallerkrankungen, in denen die Stuhlfrequenz als Folge einer Inkontinenz erhöht ist. Ein weiteres Kriterium ist ein erhöhtes Stuhlgewicht $>200 \mathrm{~g}$, dessen Bestimmung im klinischen Alltag aber eine untergeordnete Rolle spielt.
Bei einer Dauer der Beschwerden bis zu 4 Wochen spricht man von akuten, danach von chronischen Diarrhöen [1].

\section{Pathophysiologie von Durchfallerkrankungen}

Die Pathophysiologie von Durchfällen ist vielfältig. Mit der Nahrung werden pro Tag etwa 2 I Flüssigkeit aufgenommen, weitere 7-9l werden über Speicheldrüsen, den Magen, die Galle, das Pankreas und die Darmschleimhaut sezerniert. Die wesentliche Flüssigkeitsresorption erfolgt im Dünndarm, sodass nur etwa 2l/Tag in den Dickdarm übertreten und dort auf ein Stuhlvolumen von etwa $100 \mathrm{ml}$ konzentriert werden. Diesen Flüssigkeitsbewegungen liegt wesentlich ein aktiver Transport von Elektrolyten zugrunde, denen Wasser passiv nachfolgt [2].

Bei Störungen dieses Gleichgewichts kann es zu Durchfällen kommen. Beispielsweise stimuliert bei der Cholera das Choleratoxin dauerhaft GTPasen und eine nachfolgende cAMP-vermittelte Chlorid-Sekretion, die zu massiven Flüssigkeitsverlusten über den Darm führen kann. Im Rahmen entzündlicher Prozesse kommt es neben einer aktiven Flüssigkeitssekretion zudem durch Störung der Darmbarriere zu einer verminderten Resorption von Elektrolyten und Flüssigkeit. Weitere Einflussfaktoren sind neuroendokrine Botenstoffe, die Peristaltik und die bakterielle Zusammensetzung der Darmflora.

Eine osmotische Diarrhö hingegen entsteht durch unzureichend resorbierte Nahrungsbestandteile bzw. deren bakterielle Stoffwechselprodukte, die osmotisch Flüssigkeit im Darmlumen binden und daher Durchfälle begünstigen. 


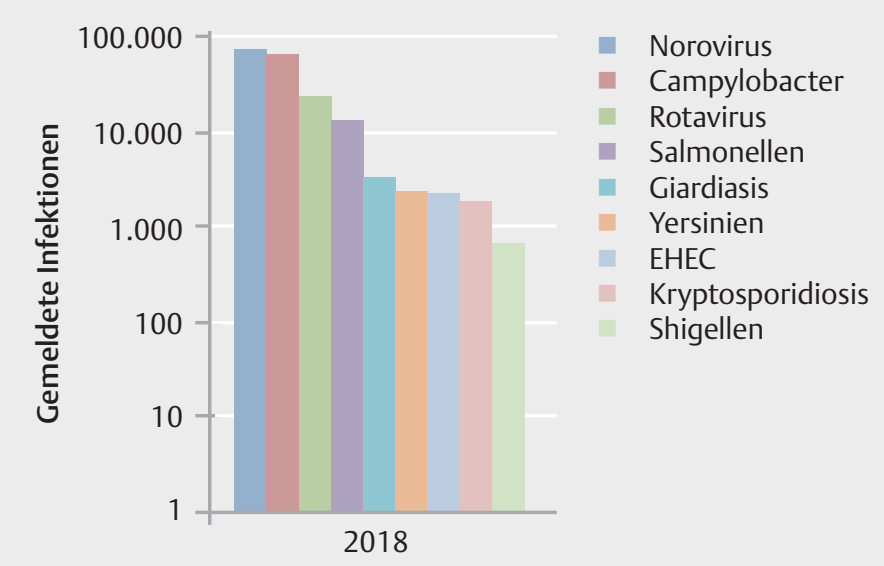

- Abb. 1 Häufigkeit der in Deutschland im Jahr 2018 gemeldeten Infektionen. Angegeben sind die bestätigten Fälle nach Referenzdefinition des Robert-Koch-Instituts [18]. EHEC = enterohämorrhagische Escherichia coli.

\section{PRAXISTIPP}

Die Unterscheidung zwischen sekretorischen/entzündlichen und osmotischen Diarrhöen ist für die klinische Differenzialdiagnose und aus didaktischen Gründen sinnvoll, im klinischen Alltag kommt es jedoch häufig zu Überlappungen.

Eine Unterscheidung zwischen sekretorischen und osmotischen Diarrhöen gelingt in der Regel bereits durch die Anamnese, da osmotische Diarrhöen nahrungsabhängig sind und daher in der Regel nicht nachts oder in Fastenphasen auftreten.

\section{Akute Diarrhö}

Die Inzidenz von akuten Durchfallerkrankungen in Deutschland beträgt etwa 1 pro Einwohner und Jahr, und die Beschwerden halten im Schnitt für 3,7 Tage an [3]. Akute Durchfallepisoden haben in der Regel eine infektiöse Genese und verlaufen selbstlimitierend. Daher ist eine Erregerdiagnostik mittels einer Stuhlprobe nur bei Patienten mit hohem Risiko erforderlich (schwerer Krankheitsverlauf oder Komorbidität) oder wenn eine Infektionsgefahr für die Umwelt besteht, z. B. im stationären Setting. Allerdings hat die Häufigkeit schwerer Infektionen besonders bei älteren Patienten in den letzten Jahren deutlich zugenommen [4]. Die Häufigkeit der in Deutschland gemeldeten Erkrankungen im Jahr 2018 ist in $>$ Abb. 1 dargestellt.

\section{Merke}

Die wichtigste therapeutische Maßnahme ist eine ausreichende Substitution von Elektrolyten und Flüssigkeit. Eine motilitätshemmende Therapie (z. B. Loperamid) sollte bei schweren Infektionen (fieberhafter Infekt, blutige Diarrhö), nicht erfolgen, da hierdurch ggf. ein Megakolon ausgelöst oder verstärkt werden kann.

\section{PRAXISTIPP \\ Meldepflicht}

Eine namentliche Meldepflicht besteht für Campylobacter, Salmonellen, Yersinia enterocolitica, enterohämorrhagische E. coli (EHEC) und sonstige darmpathogene E. coli, Shigellen, Giardia lamblia, Kryptosporidien, Rota- und Noroviren sowie bei schwerem Verlauf oder Tod durch Clostridioides difficile.

Für Cholera, Typhus und Paratyphus besteht Meldepflicht hingegen bereits bei klinisch begründetem Verdacht [5].

\section{Enteroviren}

\section{Norovirus}

Norovirus-Infektionen treten besonders in den Wintermonaten auf, betreffen alle Altersgruppen und führen zu heftigem Erbrechen sowie schweren Durchfällen. Die Inkubationszeit ist mit 24-48 h kurz, die Symptomatik bessert sich in der Regel innerhalb von 48-72 h. Die sehr hohe Kontagiosität begünstigt Ausbrüche in Heimen und Kliniken, weshalb vorbeugenden Hygienemaßnahmen eine große Bedeutung zukommt. Der Nachweis von Noroviren erfolgt mittels RT-PCR aus dem Stuhl. Die Therapie ist symptomatisch.

Bei Patienten mit Immundefekten kann es zu chronischen Norovirus-Infektionen kommen. Aufgrund einer geringen Virusausscheidung im Stuhl ist die Ansteckungsgefahr aber niedriger und es kann eine medikamentöse Therapie mit Ribavirin versucht werden.

\section{Rotavirus}

Rotaviren sind ebenfalls hochkontagiös und infizieren häufiger Kinder. Die Übertragung erfolgt fäkal-oral. Die Erkrankungsdauer beträgt 2-6 Tage, die Therapie ist symptomatisch. Ein Lebendimpfstoff steht zur Verfügung. Derzeit ist unklar, ob eine gezielte Diagnostik auf Rotaviren sinnvoll ist, da sich aus einem Virusnachweis im klinischen Alltag eigentlich keine therapeutischen Konsequenzen ergeben [5].

\section{Merke}

Erkrankungen durch andere Enteroviren sind nicht meldepflichtig und werden ebenfalls symptomatisch behandelt.

\section{Bakterielle Durchfallerreger}

Diarrhöen durch Toxin-bildende Bakterien (z. B. Toxin-bildender Staphylococcus aureus oder Bacillus cereus) sind häufig durch eine sehr kurze Inkubationszeit von wenigen 
Stunden gekennzeichnet, die Infektion erfolgt im Rahmen einer „Lebensmittelvergiftung“.

\section{Merke}

Die häufigsten bakteriellen Enteritis-Erreger sind (in absteigender Häufigkeit) Campylobacter, Salmonellen, Toxin-bildende E. coli und Shigellen.

\section{Campylobacter}

Eine Infektion mit Campylobacter erfolgt in der Regel über Lebensmittel, meist über unzureichend erhitztes Geflügel. Eine antibiotische Therapie soll nur gegeben werden, wenn es zum Zeitpunkt des Nachweises noch nicht zu einer spontanen Besserung gekommen ist oder wenn relevante Begleiterkrankungen vorliegen. Zudem ist eine Antibiotikatherapie bei Nachweis von E. coli, Campylobacter, Salmonellen und Yersinien bei Auftreten systemischer Entzündungszeichen indiziert, wozu auch Fieber und jede extraintestinale Manifestation gehören. Therapie der Wahl ist Azithromycin (z. B. 1-malig $1 \mathrm{~g}$ ) oder als 2. Wahl Ciprofloxacin (2-mal 500 mg für 3 Tage) [5].

Die Erkrankung kann durch eine reaktive Arthritis, Myokarditis oder ein Guillain-Barré-Syndrom kompliziert sein. Zudem stellen Infektionen durch Campylobacter ebenso wie durch andere Enteritis-Erreger wesentliche Risikofaktoren für ein postinflammatorisches Reizdarmsyndrom dar, das sich mit chronischen Durchfällen manifestieren kann (s. u.).

\section{Salmonellen}

Auch enteritische Salmonelleninfektionen erfolgen meist über kontaminierte Lebensmittel. Da eine antibiotische Therapie in unkomplizierten Fällen den Krankheitsverlauf nicht abkürzt, sehr wohl aber die Dauer der Salmonellenausscheidung im Stuhl verlängert, soll eine antibiotische Therapie (Ciprofloxacin 2-mal 500 mg oder Ceftriaxon $2 \mathrm{~g} /$ Tag für jeweils 5-7 Tage) nur bei Bakteriämie bzw. Zeichen einer schweren systemischen Infektion, bei Immunsupprimierten oder Dialysepatienten erfolgen [5]. Eine relative Behandlungsindikation besteht ferner bei Patienten mit Fremdkörpern/Prothesen oder Gefäßerkrankungen, da es besonders bei älteren Patienten zu einer Arteriitis bzw. Endokarditis kommen kann. Bei persistierender Ausscheidung von Salmonellen im Stuhl über einen Zeitraum von mehr als 3 Monaten kann eine antibiotische Therapie mit Ciprofloxacin für 4 Wochen versucht werden.

\section{Shigellen}

Im Gegensatz zu Campylobacter- und Salmonelleninfektionen wird bei Shigellen-Infektionen aufgrund der hohen Kontagiosität und des mitunter schweren Krankheitsverlaufs immer eine antibiotische Therapie empfohlen (z. B. Azithromycin $500 \mathrm{mg} /$ Tag für 3 Tage oder Ciprofloxacin $1 \mathrm{~g} /$ Tag für 3-5 Tage) [5].

\section{Clostridioides difficile}

Clostridioides (früher Clostridium) difficile stellen die häufigste bakterielle Ursache für im Krankenhaus erworbene (nosokomiale) Durchfallerkrankungen dar. In Deutschland treten pro Jahr etwa 100000 C.-difficile-Infektionen bei stationären Patienten auf [4]. Die hierdurch bedingte Morbidität und Mortalität haben in den letzten Jahren durch die Zunahme schwerer Begleiterkrankungen und die Verbreitung hypervirulenter Stämme zugenommen.

C. difficile kann normaler Bestandteil der Darmflora sein. Während eines Krankenhausaufenthalts ist die Wahrscheinlichkeit für eine Kolonisierung aber etwa verzehnfacht. Begünstigt wird dies durch die Tatsache, dass die Sporen resistent gegen zahlreiche Desinfektionsmittel sind. Ausgelöst wird die klinisch manifeste Infektion dann meist durch eine Störung der Darmflora (Dysbiose) nach Antibiotikatherapie. Das Risiko hierfür ist besonders durch biliär ausgeschiedene Antibiotika wie Ceftriaxon oder Ciprofloxacin erhöht und nimmt bei Immunschwäche oder Begleittherapie mit Protonenpumpeninhibitoren (PPI) weiter zu. Die häufige Verordnung von Antibiotika im ambulanten Sektor erklärt die zunehmende Bedeutung von ambulant erworbenen C.-difficile-Infektionen, die etwa 1 Viertel aller Infektionen ausmachen [6].

Die Pathogenese der C.-difficile-Infektion beruht auf der Wirkung der Toxine TcdA und TcdB, die Rho-GTPasen inaktivieren und den Zelltod der Enterozyten, Verlust der Darmbarriere und eine neutrophile Entzündungsreaktion auslösen. Das morphologische Korrelat dieser Veränderungen ist die Ausbildung charakteristischer Pseudomembranen ( $\triangleright$ Abb. 2a, b).

Schwere C.-difficile-Infektionen führen gelegentlich zu einem toxischen Megakolon, das eine Notfall-Kolektomie erforderlich macht. Bei nosokomialen Diarrhöen sollte nur eine gezielte Erregerdiagnostik auf $C$. difficile und ggf. Noroviren erfolgen. Eine Diagnostik aus geformtem Stuhl ist nicht zielführend und Laxanzien sollten mindestens $48 \mathrm{~h}$ zuvor abgesetzt werden. Derzeit gibt es keine einheitlichen Empfehlungen für den mikrobiologischen Diagnosealgorithmus. Zunächst kann ein Screeningtest auf das C.difficile-„,common-Antigen“ mittels Glutamatdehydrogenase-Test erfolgen. Da dieses Antigen aber auch von nicht toxinogenen Stämmen exprimiert wird, ist bei positivem Testergebnis als Bestätigungstest ein Toxin-Nachweis oder Zytotoxizitäts-Assay erforderlich. Aufgrund der relativen Instabilität der Toxine sollte der Stuhl gekühlt werden oder innerhalb von $2 \mathrm{~h}$ untersucht werden. Wiederholungsuntersuchungen bei initial negativem Testergebnis führen bei weniger als $5 \%$ der Fälle zu einem positiven Befund und sollten vermieden werden.

Bei klinischem Verdacht kann bereits vor Eintreffen der mikrobiologischen Befunde die Therapie begonnen werden, 

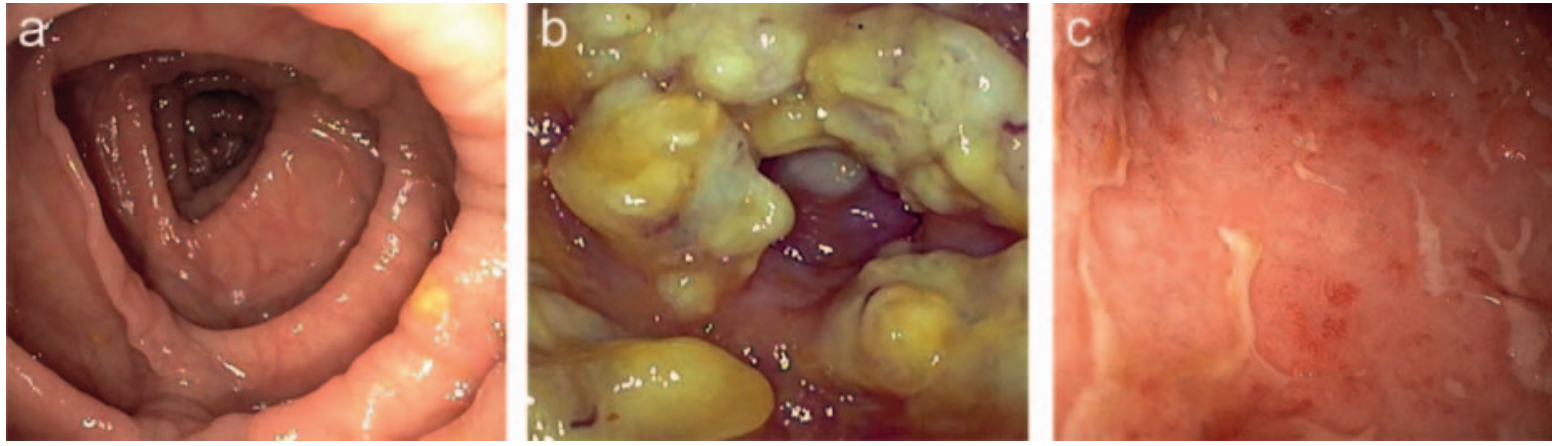

- Abb. 2 Endoskopische Kolonbefunde. a Gesundes Kolon. b Pseudomembranöse Kolitis im Rahmen einer Clostridioides-difficileInfektion. c Ulcera im Rahmen einer floriden Colitis ulcerosa.

die bei unkomplizierten Verläufen mit Metronidazol (3-mal $400 \mathrm{mg}$ per os oder 3-mal $500 \mathrm{mg}$ intravenös) bzw. bei Rezidiven oder schweren Verläufen mit oralem Vancomycin (4-mal 125-250 mg/Tag) durchgeführt wird. Die Behandlung sollte mindestens $48 \mathrm{~h}$ über das Sistieren der Durchfälle fortgeführt werden. Zudem sollten die Risikofaktoren für Rezidive, soweit möglich, minimiert werden (Absetzen von anderen Antibiotika und PPI, soweit möglich).

Mikrobiologische Verlaufsuntersuchungen im Stuhl sind nicht sinnvoll, da auch nach Ausheilung der Infektion noch bei bis zu $50 \%$ der Patienten C. difficile im Stuhl nachgewiesen werden kann. Aufgrund der Sporen kommt es allerdings häufig zu Rezidiven, die durch eine orale Vancomycin-Pulstherapie (z. B. jeweils $125 \mathrm{mg}$ Vancomycin 4-mal/Tag für 2 Wochen, dann 2-mal/Tag für 1 Woche, dann 1-mal/Tag für 1 Woche, dann 1-mal alle 2-3 Tage für 2 Wochen) oder Fidaxomicin oral behandelt werden können.

Bei rezidivierenden Verläufen kann durch einen fäkalen Mikrobiota-Transfer (FMT, „Stuhltransplantation“) sehr wirksam eine Heilung erreicht werden [6]. Andere neue Therapieansätze basieren auf der Gabe nichtresorbierbarer Betalaktamase, um die antibiotikabedingte Betalaktamasehemmung im Darm zu antagonisieren und der Entstehung einer Dysbiose vorzubeugen. Zudem können monoklonale Antikörper gegen das TcdB-Toxin zur Rezidivprophylaxe eingesetzt werden [7]; eine Impfung befindet sich in klinischer Prüfung.

\footnotetext{
Merke

Bei bakterieller Durchfallerkrankung besteht eine Indikation zur antibiotischen Therapie bei Infektionen mit Shigellen oder C. difficile, sonst nur bei Hochrisikopatienten oder systemischen Entzündungszeichen.
}

Bezüglich des Vorgehens bei Reisediarrhö wird auf entsprechende Übersichtsarbeiten verwiesen [8].

\section{Chronische Diarrhö}

Zur Differenzialdiagnose der chronischen Diarrhö kann basierend auf der Klinik eine Einteilung in sekretorische und osmotische Diarrhöen vorgenommen werden. Bei Erkrankungen, die mit intestinalen Motilitätsstörungen einhergehen (z. B. bei autonomer Neuropathie oder übermäßiger hormoneller Stimulation), spricht man von motorischer Diarrhö. Sekretorische Diarrhöen lassen sich weiter in entzündliche und nichtentzündliche Ursachen unterteilen. Eine Übersicht über die wichtigsten Differenzialdiagnosen findet sich in $\mathbf{~ A b b . ~} \mathbf{3}$.

\section{FALLBEISPIEL}

Ein 74-jähriger Patient stellt sich zur Abklärung chronischer Diarrhöen vor, die jetzt seit 6 Monaten bestehen. Als Vorerkrankung ist seit 10 Jahren ein Diabetes mellitus bekannt. Die Stuhlfrequenz beträgt derzeit 8-mal/24h (davon 6-7-mal tags, 1-2-mal nachts). Ferner leidet er an einem Gewichtsverlust von $5 \mathrm{~kg}$ in 3 Monaten und teils übelriechenden Blähungen. Wegen der auch nächtlich auftretenden Durchfälle und des Gewichtsverlusts (Alarmzeichen) erfolgt zunächst eine Abklärung auf entzündliche/sekretorische Diarrhöen. Laborchemisch ergeben sich im Blut keine relevanten Auffälligkeiten, das Calprotectin im Stuhl ist normwertig. In der Abdomensonografie zeigen sich ebenfalls keine Auffälligkeiten, wobei das Pankreas luftüberlagert ist. Auch in der Ösophagogastroduodenoskopie mit Duodenalbiopsie und Ileokoloskopie mit Stufenbiopsien ergibt sich kein pathologischer Befund.

Zunächst wird ein symptomatischer Therapieversuch mit Colestyramin und Führen eines Ernährungstagebuchs zum Erkennen von Nahrungsmittelunverträglichkeiten sowie ein Fastenversuch für 1-2 Tage und eine Wiedervorstellung nach 4 Wochen vereinbart. 


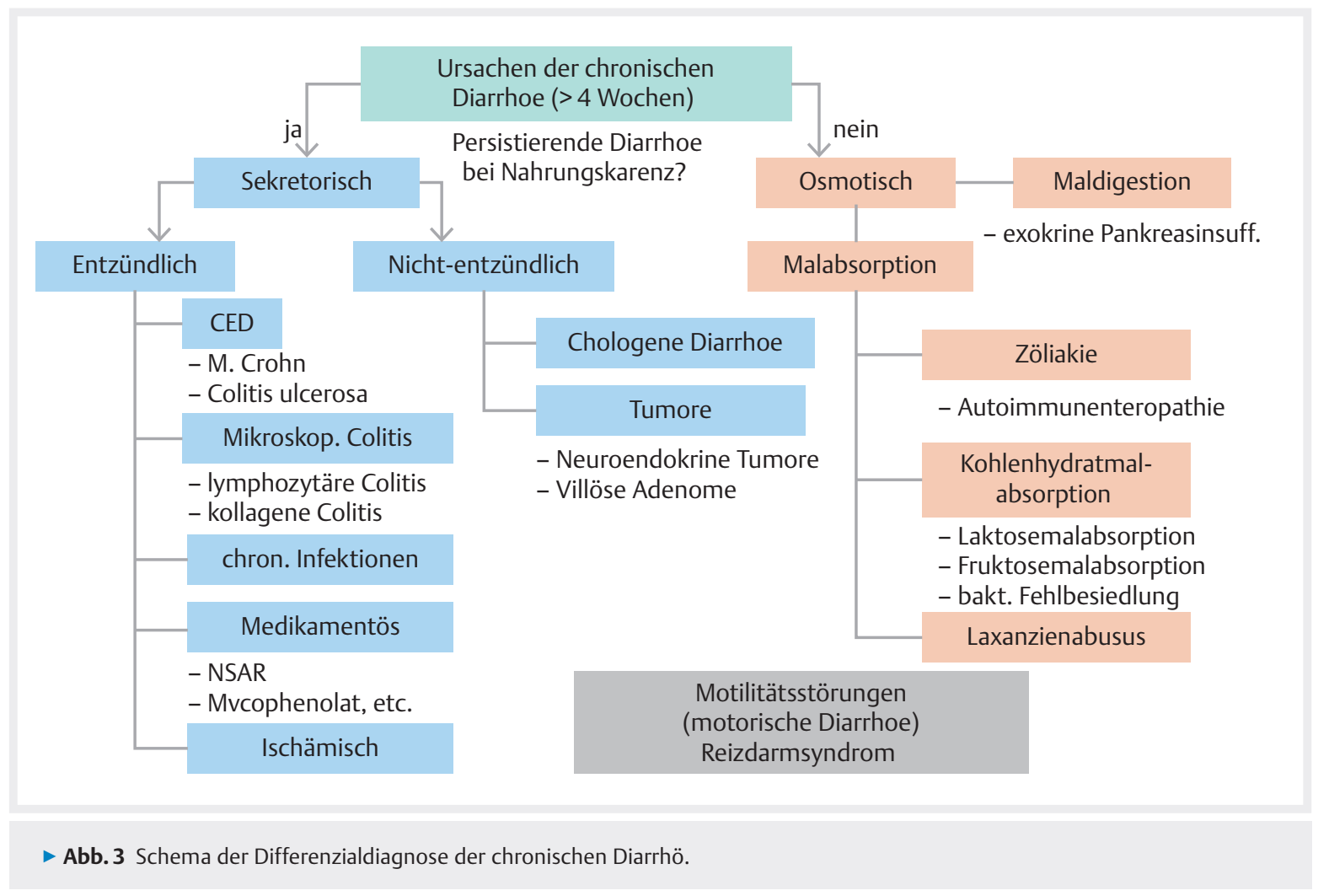

\section{Sekretorisch entzündliche Durchfälle}

Entzündliche Diarrhöen gehen meistens einher mit „Alarmzeichen“ wie

- Blut im Stuhl,

- Gewichtsverlust oder

- Fieber.

Bei Vorliegen dieser Alarmzeichen ist immer eine sorgfältige diagnostische Abklärung inkl. Endoskopie erforderlich.

\section{Merke}

Zur Diagnose bzw. zum Ausschluss entzündlicher Diarrhöen sind Entzündungsmarker im Stuhl wie Calprotectin oder Lactoferrin hilfreich. Aber auch bei normwertigem Calprotectin kann eine chronisch entzündliche Darmerkrankung vorliegen.

\section{Chronisch entzündliche Darmerkrankungen (CED)}

Etwa 400000 Patienten leiden in Deutschland an den chronisch entzündlichen Darmerkrankungen Morbus Crohn und Colitis ulcerosa, die die wichtigsten Ursachen für chronische, sekretorisch entzündliche Diarrhöen darstellen. Leitsymptome sind [9]:

- Bauchschmerzen,

- teils blutige Durchfälle und

- Gewichtsverlust.
Etwa die Hälfte der Patienten leidet zudem an extraintestinalen Manifestationen an Gelenken, Haut, Augen und den Gallenwegen.

Bei Verdacht auf eine CED sollte neben Anamnese und klinischer Untersuchung eine Laboruntersuchung (Blutbild, fäkale Entzündungsmarker wie Calprotectin, Elektrolyte, Nierenwerte, Eisenstatus, Leberwerte) durchgeführt werden. Bei Erstmanifestation und jedem Schub sollte zudem eine bakterielle Infektion (inkl. C. difficile) mittels Stuhlkultur ausgeschlossen werden.

Besonders bei Patienten mit steroidrefraktärem Verlauf sollte auch an das Vorliegen einer Zytomegalievirus (CMV)-Kolitis gedacht werden. Die Entzündungsaktivität lässt sich sonografisch und insbesondere endoskopisch objektivieren ( $\triangleright$ Abb. 2c). Die Therapie im Schub besteht zunächst aus systemischen und topischen 5-Aminosalizylaten bzw. Kortikosteroiden. Kortikosteroide sollten wegen der erheblichen Nebenwirkungen und eingeschränkten Wirksamkeit nicht zur langfristigen Remissionserhaltung eingesetzt werden. Bei steroidrefraktärem Verlauf kann eine Therapie mit TNF-Antikörpern, dem IL-12/IL-23-Antikörper Ustekinumab oder (bei Colitis ulcerosa) mit dem JAK-Inhibitor Tofacitinib bzw. Ciclosporin oder Tacrolimus erfolgen. Zur Remissionserhaltung kommen neben Thiopurinen (Azathioprin oder 6-Mercaptopurin), Methotrexat (bei Morbus Crohn), TNF-Antikörper, Ustekinumab oder Integrin-Antikörper (Vedolizumab) zum Einsatz. Alternativ sollte bei komplizierten 

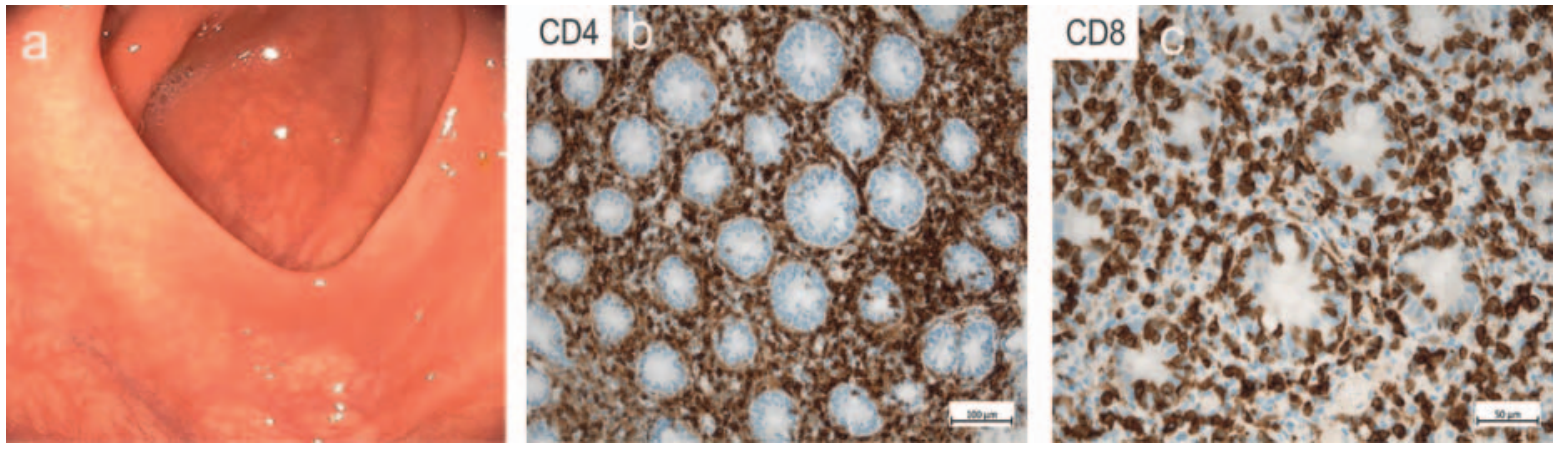

- Abb.4 Endoskopiebefund eines Patienten mit chronischer Diarrhö nach Nivolumab-Therapie eines Melanoms. Der makroskopische Befund war bis auf eine Schleimhautrötung unauffällig, histologisch zeigte sich jedoch eine ausgeprägte Infiltration durch CD4 +- bzw. CD8 +-T-Lymphozyten. a Endoskopie: unauffälliger Befund bis auf Schleimhautrötung. b Histologie: Infiltration durch CD4 +-T-Lymphozyten. c Histologie: Infiltration durch CD8 +-T-Lymphozyten.

Verläufen interdisziplinär eine etwaige OP-Indikation diskutiert werden $[9,10]$.

\section{Mikroskopische Kolitis}

Eine mikroskopische Kolitis kann sich histologisch als lymphozytäre oder kollagene Kolitis manifestieren und führt zu wässrigen sekretorischen Diarrhöen. Das mittlere Erkrankungsalter beträgt ca. 65 Jahre, wobei Frauen deutlich häufiger betroffen sind. Häufig besteht eine Assoziation der Krankheit mit einer Therapie mit nichtsteroidalen Antirheumatika (NSAR) oder PPI.

\section{Merke \\ Meist sind bei mikroskopischer Kolitis Entzündungs- marker wie Calprotectin im Stuhl oder C-reaktives Protein im Serum nicht erhöht. Die Diagnosestellung erfolgt histologisch durch Stufenbiopsien aus der makroskopisch weitgehend unauffälligen Kolon- schleimhaut.}

Zur Therapie wird Budesonid eingesetzt. Wenn es nach Absetzen oder Dosisreduktion von Budesonid zu Rezidiven kommt, kann eine remissionserhaltende Therapie in Analogie zu CED versucht werden [11].

\section{Chronische Infektionen}

Die Differenzialdiagnose für chronische Infektionen im Darm ist vielfältig. Eine Abklärung von parasitären Erkrankungen (insbesondere Giardia lamblia und Entamoeben) wird bei entsprechender Reiseanamnese in Endemiegebiete empfohlen. Andere seltene chronische infektiöse Ursachen umfassen die Darmtuberkulose, HIV-Enteropathie sowie die CMV-Kolitis bei immunsupprimierten Patienten, insbesondere unter hochdosierter Steroidtherapie.

Ein Morbus Whipple (verursacht durch eine Infektion mit Tropheryma whipplei) manifestiert sich neben einer Malabsorption im Rahmen der Zottenatrophie häufig mit extraintestinalen Symptomen wie Arthritiden oder einer kulturnegativen Endokarditis. Diese können der gastrointestinalen Symptomatik oft Jahre vorausgehen. Die Diagnose gelingt durch die Duodenalbiopsie histologisch bzw. mittels PCR. Vor Therapieeinleitung sollte mittels Liquorpunktion ein ZNS-Befall ausgeschlossen und die Therapie nach Rücksprache mit erfahrenen Zentren eingeleitet werden (z. B. Ceftriaxon $2 \mathrm{~g} /$ Tag für 2 Wochen gefolgt von 2-mal 960 mg Cotrimoxazol für 1 Jahr).

\section{Medikamentös induzierte Diarrhö}

Durchfälle machen etwa $7 \%$ aller Medikamentennebenwirkungen aus, weshalb eine sorgfältige Medikamentenanamnese erfolgen sollte. Als Ursache kommt eine osmotische (z. B. Acarbose, Laxanzien, Olmesartan-induzierte Zottenatrophie), sekretorische (z. B. Antiarrhythmika, Metformin, Mesalazin), motorische (z. B. L-Thyroxin, Irinotecan, Erythromycin) oder entzündliche (z. B. Carbamazepin, Zytostatika, NSAID) Genese in Betracht [12]. Besonders eine Therapie mit NSAR kann zu einer chronischen Entzündung im Darm und nachfolgend teils blutigen Durchfällen führen. Nach Nierentransplantation ist eine Therapie mit Mycophenolat dosisabhängig eine häufige Durchfallursache. In den letzten Jahren hat zudem die nach Checkpoint-Inhibition auftretende Autoimmunenteritis eine große Bedeutung erlangt, die sich als lymphozytäre Kolitis manifestiert ( $\vee$ Abb.4).

Die Therapie erfolgt je nach Schwere der Symptomatik zunächst mit oralen bzw. parenteralen Kortikosteroiden (0,5-1 mg/kg KG Prednison) bzw. bei unzureichendem Ansprechen mit TNF-Antikörpern oder Ciclosporin A in Analogie zur CED-Therapie [13].

\section{Ischämische Kolitis}

Die ischämische Colitis betrifft besonders ältere Patienten. Oft liegen begleitende periphere Gefäßerkrankungen vor. Als Folge einer chronischen okklusiven oder nichtokklusiven Minderperfusion kann es zu Entzündung, Bauchschmerz und blutigen Diarrhöen kommen. Die Diagnostik 
erfolgt mittels Abdomen-CT und koloskopisch. Auch den Durchfällen bei Langstreckenläufern („Runners Diarrhea“) liegt eine intestinale Minderperfusion zugrunde.

\section{Sekretorisch nichtentzündliche Durchfälle}

\section{Chologene Diarrhö}

Eine langstreckige Entzündung oder Resektion des terminalen Ileums führt zu einer unzureichenden Resorption von Gallensäuren, die im Kolon die Flüssigkeitssekretion und Motilität stimulieren und ausgeprägte sekretorische Diarrhöen auslösen können. Eine Überschreitung der Resorptionskapazität von Gallesäuren liegt wohl auch der Pathophysiologie von Diarrhöen nach einer Cholezystektomie zugrunde. Diagnostische Tests zur Messung der Gallensäureresorption stehen nur in wenigen Zentren zur Verfügung.

\section{Merke}

Bei klinischem Verdacht auf das Vorliegen einer chologenen Diarrhö kann eine empirische Therapie mit gallesäurebindenden Medikamenten (z. B. Colestyramin) initiiert werden, die meist zu einer schlagartigen Besserung der Beschwerden führt.

\section{Tumorerkrankungen}

Neuroendokrine Tumoren sind sehr seltene Auslöser sekretorischer Diarrhöen, weshalb eine entsprechende Hormondiagnostik nur erfolgen sollte, wenn keine andere Erklärung für chronische Diarrhöen gefunden werden kann. Zur Diagnostik eignet sich die Bestimmung der Serumkonzentrationen von Chromogranin A und Gastrin (Zollinger-Ellison-Syndrom, vor der Untersuchung müssen PPI mindestens 1 Woche abgesetzt werden), von vasointestinalem Peptid (VIPom, Werner-Morrison-Syndrom) bzw. die Bestimmung der 5-Hydroxyindolessigsäure im 24 h-Sammelurin zur Diagnostik eines Karzinoids. Auch Kolonkarzinome bzw. Polypen mit ausgeprägt villöser Differenzierung können chronische Diarrhöen auslösen. Maligne und gutartige Erkrankungen, die zu einer vermehrten Freisetzung von Schilddrüsenhormonen, Parathormon oder Calcitonin führen, können ebenfalls motorische Diarrhöen auslösen.

\section{Osmotische Diarrhö}

Osmotische Diarrhöen sind Folge einer Malassimilation und treten nahrungsabhängig auf.

\section{Zöliakie}

Die Zöliakie hat in Deutschland eine Prävalenz von etwa $0,3 \%$, wobei die Dunkelziffer hoch ist [14]. Pathophysiologisch kommt es bei Patienten mit entsprechendem HLA-Genotyp (DQ2 und/oder DQ8) zu einer Immunantwort gegen Gliadin bzw. das Gliadin-bindende Enzym Transglutaminase. Dies führt zu einer lymphozellulären Entzündung, Zottenatrophie und Kryptenhyperplasie ( $\triangleright$ Abb.5) sowie Bildung entsprechender Antikörper ge-
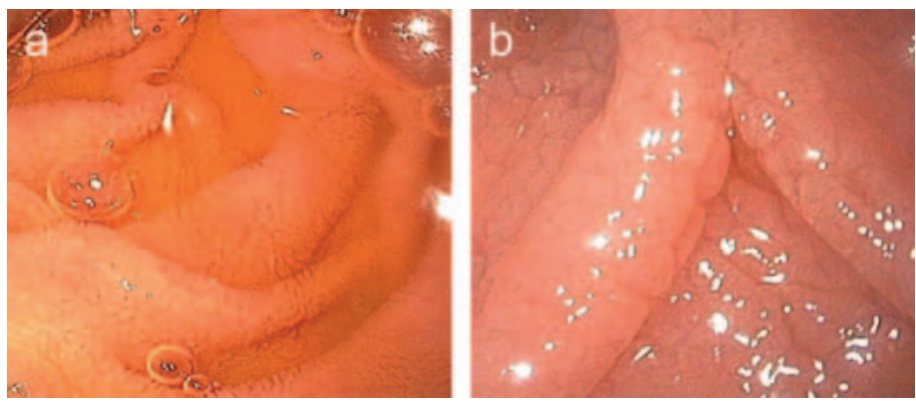

- Abb. 5 Endoskopische Darstellung der Zottenstruktur. a Regelrechte Zottenstruktur im Duodenum (links). b Die Zottenatrophie und Krypthyperplasie bei einer Patientin mit Zöliakie führt zu einem Pflastersteinrelief (rechts).

gen Transglutaminase und Gliadin. Durchfälle sind Folge der Malabsorption bei Zottenatrophie.

Da die Entzündung häufig nur auf den proximalen Dünndarm bzw. das Duodenum beschränkt ist, manifestiert sich die Erkrankung bei vielen Patienten mit einer Eisenmangelanämie als Folge der gestörten Eisenresorption. Zur Diagnostik hat der Nachweis von TransglutaminaseIgA-Antikörpern eine hohe Sensitivität und Spezifität. Da etwa 2-3\% der Patienten mit Zöliakie einen selektiven IgA-Mangel haben, muss gleichzeitig die Gesamt-IgA-Serumkonzentration bestimmt werden. Bei IgA-Mangel erfolgt die Bestimmung von IgG gegen Transglutaminase oder deamidierte Gliadinpeptide. Goldstandard der Diagnostik ist die Endoskopie mit Entnahme von mindestens 6 Duodenalbiopsien [14].

Die Therapie erfolgt durch eine strenge und lebenslange glutenfreie Diät. Die Zöliakie ist mit dem Auftreten anderer Autoimmunerkrankungen (u. a. Hashimoto-Thyreoiditis, Diabetes mellitus Typ 1) assoziiert.

\section{Merke}

Erstgradigen Verwandten von Zöliakie-Patienten sollte ebenfalls eine Zöliakie-Diagnostik angeboten werden.

Von der Zöliakie ist die Weizensensitivität (früher Glutenunverträglichkeit) abzugrenzen, die nicht immunologisch vermittelt und wohl Gluten-unabhängig ist. Vielmehr kommt es durch andere Weizenbestandteile wie die Amylase-Trypsin-Inhibitoren (ATI) über Toll-likeRezeptoren zu einer Entzündung, Barrierestörung und nachfolgenden Bauchbeschwerden. Auch die seltene Autoimmunenteropathie kann sich mit einer Malabsorption aufgrund einer Zottenatrophie manifestieren.

\section{FALLBEISPIEL}

Unter der Colestyramin-Therapie kommt es nicht zu einer nachhaltigen Besserung. Im Ernährungstagebuch lassen sich keine eindeutigen Nahrungsmittel- 
unverträglichkeiten eruieren. Sehr wohl jedoch kommt es während des Fastenversuchs zu einer deutlichen Besserung der Durchfälle, sodass diese wohl doch osmotischer Genese waren. Bei normwertigen Transglutaminase-IgA-Antikörpern und regelrechtem Gesamt-IgA ergibt sich kein Hinweis für das Vorliegen einer Zöliakie. Zudem wird ein GlukoseH2-Atemtest durchgeführt, der positiv ist.

Daher wird die Diagnose einer bakteriellen Fehlbesiedlung gestellt und eine Antibiotikatherapie mit Rifaximin durchgeführt. Die Beschwerden rezidivieren aber bereits nach 4 Wochen. In der zeitlich verzögert eingesandten Stuhlprobe zeigt sich jedoch die Elastase hochgradig supprimiert, was vereinbar mit dem Vorliegen einer exokrinen Pankreasinsuffizienz ist. In der daraufhin durchgeführten Computertomografie zeigt sich das Bild einer chronischen Pankreatitis ohne Hinweise auf das Vorliegen eines Pankreaskarzinoms. Die Beschwerden bessern sich nun rasch und anhaltend unter einer Therapie mit Pankreatin.

\section{Kohlenhydratmalabsorption}

Das häufigste Kohlenhydratmalabsorptionssyndrom ist die Laktosemalabsorption. Bei Laktasemangel kann das Disaccharid Laktose nicht in Glukose und Galaktose gespalten und resorbiert werden. Durch den bakteriellen Stoffwechsel kann es zu Blähungen und Durchfall kommen. Die Laktase-Expression nimmt mit dem Alter ab und ist genetisch determiniert. Zudem kommt es im Rahmen einer Zottenatrophie oder Entzündung im Dünndarm regelhaft ebenfalls zu einer Laktosemalabsorption. Nicht alle Menschen mit Laktosemalabsorption haben nach Verzehr von laktosehaltiger Nahrung auch Beschwerden (eine Laktoseintoleranz). Hier spielt die Schmerzwahrnehmung bei Distension des Darms eine wesentliche Rolle und die Übergänge zum Reizdarmsyndrom (s. u.) sind fließend.

Die Diagnosestellung erfolgt durch eine Nahrungsmittelanamnese („Was passiert, wenn Sie einen halben Liter Milch trinken?") bzw. einen H2-Laktose-Atemtest. Entzündungsmarker sind i.d. R. nicht erhöht. Die Therapie besteht aus einer laktosearmen Kost und ggf. der Einnahme von Laktase.

Zur Fruktosemalabsorption kommt es bei unzureichender Fruktoseresorption über den GLUT5-Transporter aufgrund einer vermehrten Fruktoseaufnahme, unzureichenden Transporterfunktion oder -expression bzw. beschleunigten Darmpassage. Der Nachweis gelingt über einen H2-Fruktoseatemtest; die Therapie erfolgt diätetisch. Eine Kohlenhydratmalabsorption kann aber auch durch eine bakterielle Fehlbesiedlung bedingt sein, die als Folge von Infekten, Operationen oder bei Darm- stenosen, z. B. im Rahmen eines Morbus Crohn, auftreten kann. Die Therapie erfolgt antibiotisch.

\section{Cave}

Bei der Diagnostik einer bakteriellen Fehlbesiedlung mittels H2-Atemtests sollte beachtet werden, dass es infolge einer beschleunigten Darmpassage bei etwa der Hälfte der Patienten zu einem falsch positiven Befund kommen kann [15].

\section{PRAXISTIPP}

Von den Nahrungsmittelunverträglichkeiten, die nicht immunologisch vermittelt sind, sind die Nahrungsmittelallergien abzugrenzen. Diese sind bei Erwachsenen selten und beruhen in der Regel auf Kreuzreaktionen gegen andere Allergene wie Pollen.

\section{Exokrine Pankreasinsuffizienz}

Osmotische Diarrhöen bei exokriner Pankreasinsuffizienz entstehen als Folge der Maldigestion bei fehlender Enzymaktivität. Dies ist häufig Folge einer chronischen Pankreatitis. Patienten berichten häufig über Durchfälle, Blähungen und Gewichtsverlust. Zur Steatorrhöe kommt es meist erst in Spätstadien der Erkrankung. Einen wichtigen Hinweis auf diese Diagnose liefert die Anamnese rezidivierender Pankreatitiden bzw. das Vorliegen entsprechender Risikofaktoren wie ein Alkohol- und Nikotinabusus.

Die Diagnose wird durch die deutlich erniedrigte Elastase im Stuhl gestellt. Dieser Test ist spezifisch für die humane Elastase und interagiert daher nicht mit oral eingenommenen Pankreatin-Präparaten, die aus Schweinepankreasextrakten hergestellt werden. Zu beachten ist, dass es bei Diarrhöen anderer Genese aber zu einem falsch positiven Testergebnis kommen kann. Goldstandard für die Diagnosestellung der exokrinen Pankreasinsuffizienz sind direkte Funktionstests wie der Sekretin-Test, der aber nur in wenigen Zentren verfügbar ist. Die Therapie erfolgt durch die Einnahme von Pankreatin.

Merke

Bei neu diagnostizierter exokriner Pankreasinsuffizienz sollten immer auch ein Pankreaskarzinom und eine endokrine Pankreasinsuffizienz (Diabetes mellitus) ausgeschlossen werden.

\section{Funktionelle Diarrhö und Reizdarmsyndrom}

Ein Reizdarmsyndrom (RDS) liegt nach den Rom-IV-Konsensuskriterien vor, wenn über einen Zeitraum von 3 Monaten mindestens 1-mal pro Woche Bauchschmerzen auftreten, die mit der Defäkation und/oder Änderungen 
der Stuhlfrequenz bzw. -konsistenz einhergehen und wenn die Beschwerden seit über 6 Monaten bestehen [16]. Andere Diagnosen sollten hinreichend ausgeschlossen sein. Bei Fehlen von Bauschmerzen spricht man von einer funktionellen Diarrhö.

\section{Merke}

Bei chronischer Diarrhö sollte immer eine eingehende diagnostische Abklärung erfolgen, bevor die Diagnose eines RDS vom Diarrhötyp gestellt wird.

Die Pathophysiologie des RDS ist multifaktoriell. Durch Umweltfaktoren wie Ernährung, eine Dysbiose und Barrierestörung im Darm kommt es zu einer milden subklinischen Entzündungsreaktion, die zu einer gestörten Funktion des autonomen und viszeralen Nervensystems führt. Hierdurch werden die Peristaltik und Schmerzwahrnehmung gestört. Ein wesentlicher Risikofaktor sind gastrointestinale Infektionen, die mit einem deutlich erhöhten Risiko einhergehen, später an einem RDS zu erkranken. Zudem kann es zu einer bakteriellen Überwucherung im Dünndarm („small intestinal bacterial Overgrowth“, SIBO) kommen, die als bakterielle Fehlbesiedlung Durchfälle begünstigt. Des Weiteren ist bei Patienten mit RDS vom Diarrhötyp häufig die Gallensäureresorption im terminalen lleum verringert, was chologene Diarrhöen begünstigt [17].

Die Therapie ist vielfältig und umfasst Ernährungsmaßnahmen (ballaststoffreiche Kost, Flohsamen), Spasmolytika bei Bauchschmerzen bzw. einen Therapieversuch mit Colestyramin. Die gestörte Schmerzwahrnehmungsschwelle kann durch trizyklische Antidepressiva korrigiert werden, die zudem eine gute antidiarrhöische Wirkung haben (z. B. Amitryptilin 25-50 mg abends). Eine Therapie mit Motilitätshemmern wie Loperamid ist ebenso möglich wie verhaltenstherapeutische Ansätze, um eine bessere Beschwerdekontrolle zu erreichen.

\section{PRAXISTIPP}

Mit den Patienten sollte frühzeitig die Möglichkeit einer funktionellen Genese der Beschwerden thematisiert und angesprochen werden, dass „negative“ Untersuchungsbefunde zu dieser Diagnose dazugehören, um Unsicherheiten und dem daraus entstehenden Wunsch nach weiterer unnötiger Diagnostik vorzubeugen. Zudem sollte einer „psychischen Stigmatisierung“ durch Erläutern der Pathophysiologie vorgebeugt und ein Kontrolltermin zur Beurteilung des Ansprechens und ggf. Anpassung der symptomatischen Therapie angeboten werden.

\section{KERNAUSSAGEN}

- Durchfall wird durch eine vermehrte Stuhlfrequenz (>3-mal/Tag) und verminderte Stuhlkonsistenz (breiig bis flüssig) definiert. Je nach Dauer der Beschwerden unterscheidet man akute $(<4$ Wochen) und chronische Diarrhöen (> 4 Wochen).

- Akute Diarrhöen haben in der Regel eine infektiöse Genese und verlaufen meist selbstlimitierend. Die Therapie ist symptomatisch.

- Eine Indikation zur Antibiotikatherapie besteht immer bei Infektionen durch Shigellen oder Clostridioides difficile bzw. sonst bei schweren Verläufen, systemischen Entzündungszeichen oder Vorliegen schwerer Begleiterkrankungen.

- Chronische Diarrhöen können nach ihrer Pathophysiologie in sekretorische bzw. entzündliche und osmotische Diarrhöen unterteilt werden. Die Unterscheidung gelingt meist anamnestisch aufgrund der Nahrungsabhängigkeit osmotischer Diarrhöen.

- Bei chronischer Diarrhö und Vorliegen von Alarmsymptomen (Infektzeichen, Gewichtsverlust, Blut im Stuhl, Bauchschmerzen) sollte immer eine eingehende Abklärung inkl. Endoskopie erfolgen.

- Bei Erstdiagnose einer exokrinen Pankreasinsuffizienz sollte ein Pankreaskarzinom ausgeschlossen werden.

\section{Wissenschaftlich verantwortlich gemäß Zertifizierungsbestimmungen}

Wissenschaftlich verantwortlich gemäß Zertifizierungsbestimmungen für diesen Beitrag ist PD Dr. med. Peter Hasselblatt, Freiburg.

\section{Interessenkonflikt}

\section{Erklärung zu finanziellen Interessen}

Forschungsförderung erhalten: nein; Honorar/geldwerten Vorteil für Referententätigkeit erhalten: ja; von einer anderen Institution (Pharma- oder Medizintechnikfirma usw.); Bezahlter Berater/interner Schulungsreferent/Gehaltsempfänger: nein; Patent/Geschäftsanteile/Aktien (Autor/Partner, Ehepartner, Kinder) an Firma (Sponsor der Veranstaltung): ja; Patent/Geschäftsanteile/Aktien (Autor/Partner, Ehepartner, Kinder) an Firma (Nicht-Sponsor der Veranstaltung): ja.

Erklärung zu nichtfinanziellen Interessen

Die Autorinnen/Autoren geben an, dass kein Interessenkonflikt besteht. 


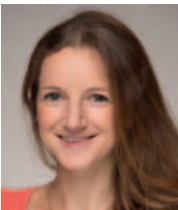

\section{Carmen Monasterio}

Dr. med., Assistenzärztin der Klinik für Innere Medizin II (Gastroenterologie, Hepatologie, Infektiologie und Endokrinologie) am Universitätsklinikum Freiburg. 2007-2013 Studium der Humanmedizin in Heidelberg. Promotion zum Thema Pankreaskarzinom am Nationalen Centrum für Tumorerkrankungen (NCT) Heidelberg. Seit 2014 in Weiterbildung zur Fachärztin für Innere Medizin und Gastroenterologie. Tätigkeit in der Darm-/CED-Ambulanz und klinische Forschung im Bereich chronisch entzündlicher Darmerkrankungen.

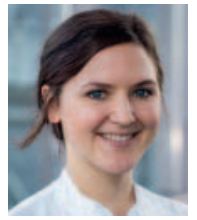

\section{Christina Hart}

Dr. med., 2010-2017 Studium der Humanmedizin an der LMU München, Promotion in der Neuroimmunologie über die Wirkung von Vitamin D bei multipler Sklerose. Seit 2017 Assistenzärztin in der Klinik für Innere Medizin II (Gastroenterologie, Hepatologie, Infektiologie und Endokrinologie) am Universitätsklinikum Freiburg. Tätigkeit in der Darm-/CED-Ambulanz und klinische Forschung im Bereich chronisch entzündlicher Darmerkrankungen.

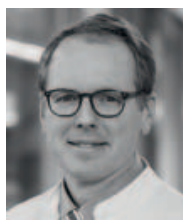

\section{Peter Hasselblatt}

PD Dr. med., Facharzt für Innere Medizin, Zusatzbezeichnung Gastroenterologie. Studium der Humanmedizin in Freiburg und Lyon. Seit 2013 Leitender Oberarzt an der Klinik für Innere Medizin II des Universitätsklinikums Freiburg und Leiter der Darm-/CED-Ambulanz. Forschungsschwerpunkte: klinische und immunologische Fragestellungen bei chronisch entzündlichen Darmerkrankungen, Signaltransduktion bei entzündlichen Lebererkrankungen und dem hepatozellulären Karzinom.

\section{Korrespondenzadresse}

PD Dr. med. Peter Hasselblatt

Klinik für Innere Medizin II

Universitätsklinikum Freiburg, Medizinische Fakultät,

Albert-Ludwigs-Universität Freiburg

Hugstetter Straße 55

79106 Freiburg

peter.hasselblatt@uniklinik-freiburg.de

\section{Literatur}

[1] Schiller LR, Pardi DS, Sellin JH. Chronic diarrhea: diagnosis and management. Clin Gastroenterol Hepatol 2017; 15: 182-193, e183. doi:10.1016/j.cgh.2016.07.028

[2] Camilleri M, Sellin JH, Barrett KE. Pathophysiology, evaluation, and management of chronic watery diarrhea. Gastroenterology 2017; 152: 515-532, e512. doi:10.1053/ j.gastro.2016.10.014

[3] Wilking $\mathrm{H}$, Spitznagel $\mathrm{H}$, Werber $\mathrm{D}$ et al. Acute gastrointestinal illness in adults in Germany: a population-based telephone survey. Epidemiol Infect 2013; 141: 2365-2375. doi:10.1017| S0950268813000046
[4] Lynen Jansen P, Stallmach A, Lohse AW et al. Entwicklung infektiöser Durchfallerkrankungen zwischen den Jahren 2000 und 2012. Z Gastroenterol 2014; 52: 549-557. doi:10.1055/ s-0033-1356442

[5] Hagel S, Epple H], Feurle GE et al. S2k-Leitlinie „Gastrointestinale Infektionen und Morbus Whipple“. Z Gastroenterol 2015; 53: 418-459. doi:10.1055/s-0034-1399337

[6] Guery B, Galperine T, Barbut F. Clostridioides difficile: diagnosis and treatments. BMJ 2019; 366: |4609. doi:10.1136/ bmj.14609

[7] Wilcox MH, Gerding DN, Poxton IR et al. Bezlotoxumab for prevention of recurrent clostridium difficile infection. $N$ Engl ] Med 2017; 376: 305-317. doi:10.1056/NEJMoa1602615

[8] Steffen R, Hill DR, DuPont HL. Traveler's diarrhea: a clinical review. JAMA 2015; 313: 71-80. doi:10.1001/jama.2014.17006

[9] Teich N, Klugmann T. Chronisch-entzündliche Darmerkrankungen: Diagnostik und Therapie in der Praxis. Dtsch Med Wochenschr 2018; 113: 945-952. doi:10.1055/a-0538-3715

[10] Preiss JC, Bokemeyer B, Buhr H] et al. Aktualisierte S3-Leitlinie „Diagnostik und Therapie des Morbus Crohn“ 2014. Z Gastroenterol 2014; 52: 1431-1484. doi:10.1055/s-0034-1385199

[11] Miehlke S, Verhaegh B, Tontini GE et al. Microscopic colitis: pathophysiology and clinical management. Lancet Gastroenterol Hepatol 2019; 4: 305-314. doi:10.1016 S2468-1253(19)30048-2

[12] Philip NA, Ahmed N, Pitchumoni CS. Spectrum of drug-induced chronic diarrhea. J Clin Gastroenterol 2017; 51: 111-117. doi:10.1097/MCG.0000000000000752

[13] Haanen J, Carbonnel F, Robert C et al. Management of toxicities from immunotherapy: ESMO Clinical Practice Guidelines for diagnosis, treatment and follow-up. Ann Oncol 2017; 28: iv119-iv142. doi:10.1093/annonc/mdx225

[14] Felber ], Aust D, Baas S et al. Ergebnisse einer S2k-Konsensuskonferenz der Deutschen Gesellschaft für Gastroenterologie, Verdauungs- und Stoffwechselerkrankungen (DGVS) gemeinsam mit der Deutschen Zöliakie-Gesellschaft (DZG) zur Zöliakie, Weizenallergie und Weizensensitivität. Z Gastroenterol 2014; 52: 711-743. doi:10.1055/s-0034-1366687

[15] Lin EC, Massey BT. Scintigraphy demonstrates high rate of false-positive results from glucose breath tests for small bowel bacterial overgrowth. Clin Gastroenterol Hepatol 2016; 14: 203-208. doi:10.1016/j.cgh.2015.07.032

[16] Mearin F, Lacy BE, Chang L et al. Bowel disorders. Gastroenterology 2016; 150: 1393-1407. doi:10.1053 j.gastro.2016.02.031

[17] Aziz I, Mumtaz S, Bholah $\mathrm{H}$ et al. High prevalence of idiopathic bile acid diarrhea among patients with diarrhea-predominant irritable bowel syndrome based on Rome III criteria. Clin Gastroenterol Hepatol 2015; 13: 1650-1655, e1652. doi:10.1016/j.cgh.2015.03.002

[18] Robert-Koch-Institut - RKI. Infektionsepidemiologisches Jahrbuch meldepflichtiger Krankheiten für 2018. Berlin: Robert Koch Institut. 2019. Im Internet (Stand: 15.04.2020): https:// www.rki.de/DE/Content/Infekt/Jahrbuch/Jahrbuecher/2018. html

\section{Bibliografie}

DOI https://doi.org/10.1055/a-0944-8523

Dtsch Med Wochenschr 2020; 145: 1325-1336

(c) Georg Thieme Verlag KG, Stuttgart · New York

ISSN 0012-0472 


\section{Punkte sammeln auf CME.thieme.de}

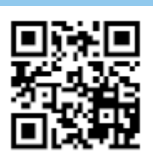

Diese Fortbildungseinheit ist in der Regel 12 Monate online für die Teilnahme verfügbar.

Den genauen Einsendeschluss finden Sie unter https://eref.thieme.de/CXDCFHU.

Sollten Sie Fragen zur Online-Teilnahme haben, finden Sie unter https://cme.thieme.de/hilfe

eine ausführliche Anleitung. Wir wünschen viel Erfolg beim Beantworten

der Fragen!

Unter https://eref.thieme.de/CXDCFHU oder über den QR-Code kommen Sie direkt zur Startseite des Wissenstests.

VNR 2760512020158723599

\section{Frage 1}

Welches sind in Deutschland die häufigsten bakteriellen Erreger von Durchfallerkrankungen?
A Shigellen
B Listerien
C Vibrio cholerae
D Campylobacter
E Kryptosporidien

\section{Frage 2}

Eine 24-jährige Patientin stellt sich wegen Bauchschmerzen, Blähungen, leichten Durchfällen nach der Nahrungsaufnahme und Gewichtsverlust $(5 \mathrm{~kg}$ ) beim Hausarzt vor. Die Beschwerden bestehen seit 4 Monaten, eine Eisenmangelanämie ist gesichert. Welche Aussage ist richtig?

A Eine Zöliakie sollte ausgeschlossen werden.

B Aufgrund der typischen Klinik und des Alters liegt am ehesten ein Reizdarmsyndrom vom Diarrhötyp vor, weshalb keine weitere Diagnostik erforderlich und eine symptomatische Therapie ausreichend ist.

C Die Klinik passt gut zu einer chologenen Diarrhö, eine Colestyramin-Therapie ist indiziert.

D Eine möglicherweise zugrunde liegende Laktoseintoleranz sollte durch Allergietests gegen Milcheiweiß (IgE im Serum und Prick-Test) ausgeschlossen werden.

E Die Beschwerden lassen sich am ehesten auf eine chronische gastrointestinale Infektion (z. B. Campylobacter-Dauerausscheiderin) zurückführen.

\section{Frage 3}

Wodurch ist die Clostridioides-difficile-assoziierte Enteritis gekennzeichnet?

A Clostridioides difficile ist eine neue, aggressivere Unterart von Clostridium difficile.

B Ein 86-jähriger Patient erkrankt während eines stationären Aufenthalts wegen einer Pneumonie an Durchfällen, die für mehrere Tage anhalten. Als mikrobiologische Stuhldiagnostik sind zunächst Nachweisverfahren für $C$. difficile bzw. dessen Toxine ausreichend.

C Eine Begleittherapie mit Protonenpumpenhemmern mildert den klinischen Verlauf.

D Mikrobiologische Verlaufsuntersuchungen sind It. Leitlinienempfehlungen bei jeder gesicherten Clostridioides-difficileassoziierten Enteritis angezeigt.

E Während eines Krankenhausaufenthalts ist die Wahrscheinlichkeit für eine Kolonisierung mit $C$. difficile doppelt so hoch wie unter normalen Umständen in einer gesunden Population.

\section{Frage 4}

Bei welcher erregerassoziierten Enteritis besteht eine Meldepflicht bereits bei klinischem Verdacht?

A enterohämorrhagische Escherichia coli (EHEC)

B Norovirus

C Paratyphus

D Rotavirus

E Shigellen

\section{Frage 5}

Welche der folgenden Durchfallerkrankung führt klinisch klassischerweise nicht zu einer sekretorischen Diarrhö?
A Cholera
B chologene Diarrhö
C VIPom
D Zöliakie
E Karzinoid

- Weitere Fragen auf der folgenden Seite... 


\section{Punkte sammeln auf CME.thieme.de}

\section{Frage 6}

Welches Testverfahren ist für ein Screening auf Zöliakie geeignet?
A intrakutaner Prick-Test gegen Gliadin
B Anti-Enterozyten-Antikörper
C Transglutaminase-IgA-Antikörper und Gesamt-IgA
D Koloskopie
E Dünndarmsonografie

\section{Frage 7}

Welche Therapie ist bei der Behandlung der C.-difficile-Infektion unwirksam?
A Vancomycin intravenös
B Metronidazol oral
C fäkaler Mikrobiota-Transfer
D Metronidazol intravenös
E Fidaxomicin oral

\section{Frage 8}

Welche Aussage zum Reizdarmsyndrom trifft zu?

A Da das Reizdarmsyndrom klassischerweise eine psychiatrische Erkrankung ist, sollte zunächst eine antidepressive Therapie erfolgen.

B Patienten mit infektiöser Enteritis haben ein deutlich erhöhtes Risiko, im weiteren Verlauf an einem Reizdarmsyndrom zu erkranken.

C Die analgetische Therapie sollte zunächst mit nichtsteroidalen Antiphlogistika erfolgen.

D Die Diagnose darf erst gestellt werden, wenn wirklich alle Differenzialdiagnosen überprüft wurden („Ausschlussdiagnose“).

E Die Klassifikation des Reizdarmsyndroms erfolgt nach den Milan-Kriterien.

\section{Frage 9}

Welche Aussage zu chronischen Durchfallerkrankungen trifft zu?

A Die durch Checkpoint-Inhibitoren ausgelöste Autoimmunkolitis ist wesentlich durch eine neutrophile Entzündungsreaktion gekennzeichnet.

B Die Therapie der durch Checkpoint-Inhibitoren ausgelösten Autoimmunkolitis erfolgt je nach Grad der Toxizität durch Kortikosteroide oder TNF-Antikörper.

C Die Behandlung einer mikroskopischen Kolitis erfolgt in Analogie zur Colitis ulcerosa primär mit Mesalazin.

D Die ischämische Kolitis tritt klassischerweise vor dem 20. Lebensjahr auf.

E Die Therapie der chronischen Zytomegalievirus-assoziierten Kolitis sollte mit Prednison erfolgen.

\section{Frage 10}

Welche Durchfallerkrankung geht typischerweise nicht mit einem erhöhten Calprotectin-Wert im Stuhl einher?
A Campylobacter-Enteritis
B Laktoseintoleranz
C Morbus Crohn
D Colitis ulcerosa
E Salmonellenenteritis 